Für jeden, der sich aktuell über Fragen des Extremismus in Deutschland und darüber hinaus informieren will, ist das neue Jahrbuch unverzichtbar. Der angezeigte Sammelband kann als aktuelle Einführung in die Extremismusproblematik gelesen werden. Eine systematisch überzeugendere Gliederung wäre allerdings möglich gewesen. Auch bleibt eine möglichst präzise Verwendung von Begriffen Wunsch des Rezensenten: Entgegen journalistischer Übung sollte nicht von „SPD-Bürgermeistern“, nicht von „Hochburgen“ nirgendwo gesellschaftlich tonangebender Kleinparteien und im Falle vorhandener parlamentarischer Stützfraktionen nicht von bloß „tolerierten“ Minderheitsregierungen die Rede sein. Aber diese weitgehend formalen Anmerkungen tun dem Wert der vermittelten Informationen keinen Abbruch.

Jürgen Plöhn

\title{
Rechtsextremismus in Deutschland und Europa: wissenschaftliche Analysen und journalistische Beobachtungen
}

Brandstetter, Marc: Die NPD unter Udo Voigt. Organisation, Ideologie, Strategie, Nomos Verlagsgesellschaft, Baden-Baden 2013, 402 Seiten, € 59,-.

Staud, Toralf und Johannes Radke: Neue Nazis. Jenseits der NPD: Populisten, Autonome Nationalisten und der Terror von rechts, Kiepenheuer \& Witsch, Köln 2012, 272 Seiten, € 9,99.

Langebach, Martin und Andreas Speit: Europas radikale Rechte. Bewegungen und Parteien auf Straßen und in Parlamenten, Orell Füssli-Verlag, Zürich 2013, 287 Seiten, € 21,95.

Bei der Kampfkandidatur um den Bundesvorsitz der „Nationaldemokratischen Partei Deutschlands“ (NPD) setzte sich beim Parteitag 2011 Holger Apfel gegen den bisherigen Amtsinhaber Udo Voigt durch. Damit ging für die rechtsextremistische Partei eine Ära zu Ende, nachdem sie zwischen 1996 und 2011 nach einem jahrzehntelangen Schattendasein erstmals wieder Erfolge bei Landtagswahlen hatte verbuchen können. Darüber hinaus entwickelte sich die NPD im rechtsextremistischen Lager zu einer dominierenden Kraft, die mit ihrem „Staubsauger“-Effekt die unterschiedlichsten Akteure anzog. Der Frage, wie in der Rückschau die „Ära Voigt“ der Partei eingeschätzt werden kann, widmet sich die umfangreiche Studie des Politologen Marc Brandstetter. Darin geht es um die Besonderheiten, die in der Entwicklung von der „alten“ NPD vor 1996 und der „neuen“ NPD nach 1996 zum Ausdruck kommen und nach deren Einfluss auf die begrenzten Erfolge der Partei.

Nach der Einleitung mit Ausführungen zur Problemstellung und einem Definitionskapitel zum politischen Extremismus beschreibt der Autor zunächst die Geschichte der NPD in den früheren Phasen des Aufstiegs von 1964 bis 1972 und des Niedergangs von 1972 bis 1996. Hierzu liegen mittlerweile ausführliche politikwissenschaftliche Studien vor. Brandstetter will sie bezogen auf den Zeitraum 1996 bis 2011 fortschreiben. Dazu geht er ausführlich auf die Person von Udo Voigt ein, der als geschickter Organisator des Wandlungsprozesses eine wichtige Rolle spielte. Die Mitglieder und die Struktur stehen danach im Zentrum des Interesses, wobei man selbst zu scheinbar eher marginalen Aspekten wie den Finanzen der Partei ausführliche Informationen erhält. Auch Absplitterungen und 
Machtkämpfe erörtert Brandstetter ausführlich. Es handele sich nicht um „eine heterogene Partei“. Viele Strömungen des Rechtsextremismus hätten dort eine Heimat gefunden. „Außerdem bestehen zwischen führenden Kadern persönliche Fehden ..." (S. 347).

Besonders ausführlich widmet sich der Autor den Wahlen, dokumentiert die Ergebnisse wie die Zusammensetzung der Wählerschaft. Hinsichtlich der Ideologie sieht er in der völkischen Deutung der „Volksgemeinschaft“ das Fundament (S. 270) und interpretiert die NPD als „Anti-Partei“ (S. 279), wobei dies mit der Negativ-Ausrichtung an Feindbildern wie „Anti-Amerikanismus“ oder „Anti-System“ erklärt wird; und schließlich geht es noch um die strategischen Grundlagen der Partei und den öffentlichen Umgang mit ihr. Danach unternimmt Brandstetter den angekündigten systematischen Vergleich von „alter“ und „neuer" NPD bezogen auf Ideologie, Organisation, Strategie und Wahlen. Hierbei lassen sich in der Tat bedeutende Änderungen in den unterschiedlichsten Bereichen ausmachen: „Soziodemographisch war die ,Thielen/ Thadden-NPD' eine Partei des ,alten Mittelstandes'. "Das Bildungsbürgertum war über-, die Arbeiterschaft unterrepräsentiert. Und weiter heißt es: „Heute stellen Arbeiter und Angestellte mehr als zwei Drittel der NPD-Basis“ (S. 346).

Brandstetter legt mit seiner Arbeit eine wichtige Ergänzung zur bisherigen Forschung über die NPD vor. Darin findet man ausführliche Daten und Informationen zu den wichtigsten Aspekten der Partei, die auch aufgrund der guten Strukturierung für den Handbuchcharakter des Bandes sprechen. Hier und da hätte man sich ein Mehr an Analyse und Einschätzung gewünscht. Gleichwohl findet man auch immer wieder entsprechende Ausführungen, wofür insbesondere der beachtenswerte Vergleich von „alter“ und „neuer“ NPD steht. Dabei arbeitet der Autor überzeugend die Besonderheiten der Voigt-NPD heraus, etwa eine Verschärfung der Extremismusintensität hin zu einem recht deutlichen öffentlichen Bekenntnis zur Ablehnung des „Systems“. Auch reproduziert Brandstetter nicht unkritisch die Selbstdarstellung der NPD hinsichtlich ihrer Präsenz in der „Mitte der Gesellschaft“. Kurzum, die kommende Beschäftigung mit der Partei hat mit Brandstetters Buch ein solides Grundlagenwerk erhalten.

Die Rechtsextremisten in Deutschland sind zwar in ihrer Ideologie vergangenheitsbezogen, gleichwohl orientieren sie sich in Agitationstechnik, Handlungsstil und Organisationsform durchaus an der Gegenwart. Darauf verweisen die Journalisten Johannes Radke, der für den „Tagesspiegel“ und „Zeit Online“ arbeitet, und Toralf Staud, der durch sein Buch „Moderne Nazis“ (2005) bekannt geworden ist. In ihrem hier anzuzeigenden gemeinsamen Werk konstatieren sie: „Der rechte Rand hat sich seit den Neunzigerjahren tiefgreifend gewandelt: er hat sich zugleich radikalisiert und verbürgerlicht" (S. 8). Diesen Entwicklungsprozess wollen die beiden Autoren in sieben Kapiteln zu unterschiedlichen Aspekten dieses politischen Lagers nachzeichnen. Das geschieht in Form von journalistischen Reportagen zu einzelnen Phänomenen, die auch unabhängig voneinander als je eigenständige Texte gelesen werden können.

Zunächst machen Radke und Staud am Beispiel von Dortmund deutlich, dass die alltagskulturelle Präsenz von Neonazis kein ostdeutsches Phänomen ist. Die Ruhrmetropole steht bei ihnen als Beispiel dafür, „was passiert, wenn gut organisierte Neonazi-Kader eine Stadt zu erobern versuchen" (S. 17). Dem folgt ein historischer Rückblick, denn die Autoren meinen den aktuellen Rechtsextremismus nur im Lichte der Entwicklung der Szene in den 1990er Jahren in Ostdeutschland verständlich machen zu können. Danach behandeln sie die „Autonomen Nationalisten“ als Form von „Patchwork-Nazis“, mache doch die Nachahmung von Habitus und Kleidung linksextremistischer Autonomer unter Beibehal- 
tung der Ideologie deutlich, „wie sehr sich der Rechtsextremismus im letzten Jahrzehnt gewandelt hat" (S. 76). Ähnliches wollen sie dann anhand der NPD und ihrer eher misslungenen Versuche zur Modernisierung aufzeigen. Gleichwohl gelang es der Partei in bestimmten Regionen Ostdeutschlands wie etwa in Vorpommern, alltagskulturell einen Machtfaktor darzustellen.

Als lagerinterner Konkurrent im Westen gelten die regionalen Ableger der „Pro Deutschland“-Partei, die mit fremden- und islamfeindlicher Propaganda auf sich aufmerksam machten. Auch wenn es an entscheidenden Wahlerfolgen mangele, vergifte diese mit das gesellschaftliche Klima. Danach gehen die Autoren ausführlicher auf die Entwicklung des Rechtsterrorismus ein, die in den Serienmorden des „Nationalsozialistischen Untergrundes“ gipfelte. Indessen halten sie dessen Agieren für Gefahren der Vergangenheit. Für die Gegenwart müsse vielmehr auf die geplante Gewaltorientierung der „Autonomen Nationalisten“ verwiesen werden: „Was diese jungen, radikalen Aktivisten so gefährlich macht, sind ihre fehlenden Skrupel Gewalt gegenüber“ (S. 222). Wenn man zukünftig Rechtsterrorismus verhindern wolle, sollte man diese Szene besonders intensiv beobachten. Das Buch endet mit einem informativen Interview mit einem Aussteiger aus der Szene und zehn Tipps der Autoren zum Umgang mit Rechtsextremisten.

Der Band zeichnet sich durch gute Lesbarkeit aus und beschreibt anschaulich die Wandlung der rechtsextremistischen Szene. Dabei schreiben die Autoren mit großer Sachkenntnis und ohne billige Polemik. Immer wieder formulieren sie auch analytisch interessante Aussagen, wozu etwa die Gefahrenprognose in Richtung der „Autonomen Nationalisten“ zählt. Nur in wenigen Fällen findet man Fehleinschätzungen: So agierten die beiden neonazistischen Polizistenmörder Michael Berger und Kay Diesner keineswegs nach dem „Lone Wolf“-Modell (S. 205). Vielmehr wollten sie sich jeweils der Verhaftung entziehen. „Hans Westmar“ (S. 207) ist auch keine reale Person, sondern eine Figur aus einem NS-Propagandafilm, die Neonazis als Pseudonym diente. Doch solche kleineren Mängel schmälern nicht die unverkennbaren Vorzüge des Buches, das sich von manchen journalistischen Darstellungen zum Thema wohltuend positiv abhebt. Auch in analytischer Hinsicht erhält der Leser immer wieder reflexionswürdige Anregungen.

Nicht nur in Deutschland, sondern allgemein in Europa lassen sich unterschiedliche Phänomene am „rechten Rand“ des politischen Spektrums ausmachen: Sie reichen von Erfolgen für rechtsextremistische oder rechtspopulistische Parteien über islam- und muslimenfeindliche Kampagnen angeblicher Bürgerbewegungen bis zu fremden- und minoritätenfeindlichen Gewaltakten. Auf die Dimension der damit einhergehenden Gefahren wollen der Sozialpädagoge Martin Langebach und der Journalist Andreas Speit aufmerksam machen. 2012 reisten sie fast das ganze Jahr über durch Europa und erlebten die radikale Rechte „vor Ort, auf der Straße, bei Kundgebungen, während Wahlkampf-Abendveranstaltungen, in Parlamentssitzungen und bei RechtsRock-Events in elf europäischen Ländern“ (S. 13). Davon berichten sie in ihrem hier anzuzeigenden Buch.

Begrifflich orientieren sich Langebach und Speit an der Definition von Michael Minkenberg, wonach der Terminus „Rechtsradikalismus“ für eine „ultranationalistische Vorstellung" stehe, die nicht notwendigerweise aktuell direkt und explizit gegen die liberale Demokratie gerichtet sei (vgl. S. 13). Damit umfasst die Darstellung ganz unterschiedliche Phänomene aus diesem politischen Bereich, wobei es um die Erfolge der NPD und die Serienmorde des NSU in Deutschland ebenso geht wie um die Neuausrichtung des „Front National“ und die Wirkung der Neuen Rechten in Frankreich, die Entwicklung der post- 
faschistischen „Alleanza Nazionale“ und die Positionen der pro-faschistischen „Casa Pound“ in Italien. So nehmen die Autoren politische Prozesse in vielen Ländern aus der journalistischen Perspektive in den Blick, womit gemeint ist, dass persönliche Eindrücke, Beobachtungen und Hintergrundgespräche mit Experten zu den jeweiligen Themen den Kernbestand der einzelnen Länderkapitel ausmachen.

Bilanzierend heißt es: „Vierzehn europäische Länder, ein internationaler Ort. Die radikale Rechte bemüht sich, gesellschaftliche Akzeptanz zu gewinnen. Auf den Straßen protestieren Parteien und Kameradschaften gegen Sozialkürzungen und Rentenaltererhöhung. In den Parlamenten, auf Landes-, nationaler oder europäischer Ebene, lehnen die Abgeordneten und Landtagsmandatsträger Einwanderung und Bankenunterstützung $a b$, und bei Konzerten beklagen Bands und Fans den Bau von Moscheen und Identitätsverluste. Themen nicht nur am rechten Rand der Gesellschaften in Europa. In den vergangenen Jahren sind in den Staaten, so belegen es Studien, rechte Ressentiments gewachsen. Die Themen der radikalen Rechten sind die Themen der nervösen Gesellschaften ...“ (S. 269) „So unterschiedlich die Parteien und Kameradschaften in den Ländern erscheinen - ihnen allen gemein ist, die Ängste und Sorgen der Bürger auszunutzen, um Zuspruch und Wählergunst zu gewinnen" (S. 270). Einschlägige Einstellungen und ökonomische Krisenerfahrungen erklärten dies.

Das Urteil über das Buch ist abhängig vom Erwartungshorizont des Lesers: Wer eine gut lesbare eindringliche Darstellung zu Entwicklungen am „rechten Rand“ in den europäischen Ländern lesen möchte, ist hier mehr als nur gut bedient. Wer eine detaillierte Analyse mit genaueren Einschätzungen erwartet, wird wohl enttäuscht sein. Es handelt sich eben um ein journalistisches Buch. Da fangen Kapitel mit Sätzen wie „Der Saal ist kühl. Die Abendsonne wirft, nach einem sehr warmen Tag, noch einige Strahlen durch die Tür“ (S. 83) an. Es fehlt an genauen Belegen wie an näheren Informationen zu einzelnen Organisationen. Gleichwohl informiert der Band auch zur Entwicklung in Ländern im Umbruch wie Griechenland oder Ungarn, woraus aus der vergleichenden Perspektive etwas gelernt werden kann. Die Darstellung bleibt aber fast nur auf dieser phänomenologischen Ebene stehen. Die Frage nach der gesellschaftlichen Wirkung wird allzu einfach mit dem Hinweis auf andere Studien beantwortet.

Armin Pfahl-Traughber

\section{Rechte Leute von links: historisch-biografische Einblicke und romanhafte Beschreibungen}

Seitenbecher, Manuel: Mahler, Maschke \& Co. Rechtes Denken in der 68er-Bewegung?, Verlag Ferdinand Schöningh, Paderborn 2013, 557 Seiten, € 39,90.

Carini, Marco: Die Achse der Abtrünnigen. Über den Bruch mit der Linken, Rotbuch Verlag, Berlin 2012, 286 Seiten, € 14,95.

Das „Zeitalter der Extreme“ (Eric Hobsbawm) spiegelt sich nicht allein in der jüngeren Geschichte des gesamten europäischen Kontinents wider, sondern bisweilen auch in den Biografien Einzelner. Einen Teil von ihnen nimmt der Band „Mahler, Maschke \& Co.“ in den 